\title{
Photoelastic measurements of polymer insert stress in the knee prostheses designed for high/deep flexion
}

\author{
A. Lawi ${ }^{\mathrm{a}}$, J. Takiguchi ${ }^{\mathrm{a}}$, M. Hasegawa ${ }^{\mathrm{a}}$, S. Hirokawa ${ }^{\mathrm{a} *}$, M. Todo $^{\mathrm{b}}$ and T. Hotokebuchi ${ }^{\mathrm{c}}$ \\ ${ }^{a}$ Faculty of Engineering, Kyushu University, Fukuoka, Japan; ${ }^{b}$ Research Institute of Applied Mechanics, Kyushu University, Fukuoka, \\ Japan; ' School of Medicine, Saga University, Saga, Japan
}

(Received 29 July 2009; final version received 31 March 2010)

\begin{abstract}
This paper presents the experimental results of photoelasticity for determining the magnitude and distribution of stresses on the polyethylene insert of the knee prostheses designed for high/deep knee flexion. The prostheses used in our experiment were a conventional posterior stabiliser knee, Scorpio NRG (Non-Restricted Geometry, Stryker Co., USA) and CFK (Complete Flexion Knee, Japan Medical Material Co., Japan), which we have developed to enable the patient to make a complete knee flexion (i.e. seiza in Japanese). Prior to the photoelastic experiment, we had introduced the forces exerted on the knee joint during ascending from deep knee flexion. Here we took squatting as an example of deep knee flexion instead of seiza because ascending from seiza contains complex modalities. The introduced data in terms of knee angles and joint forces were applied to the photoelastic models. The results demonstrated that after the knee angle became larger than $90^{\circ}$, the shear stresses on the post and cam of NRG were higher than those on the ball and socket of CFK. We conclude that the design and the configuration of CFK is acceptable at deep knee flexion from a load-bearing viewpoint.
\end{abstract}

Keywords: knee prosthesis; photoelasticity; post and cam; ball and socket; contact stress

\section{Introduction}

The objective of this study is to determine the magnitude and distribution of stresses on the polyethylene insert of knee prostheses designed for high/deep knee flexion. Current innovation in knee prosthetic design has led to such a breakthrough that knee flexion up to $150^{\circ}$ can be achieved. To attain deep knee flexion is crucial for Asian and Moslem people because their lifestyle or religious activities necessitate sitting on the floor with complete knee flexion, which is called seiza in Japanese (Akagi 2005; Thambyah et al. 2005), as shown in Figure 1(a). Even for the Western people, attaining high/deep knee flexion [Figures 1(b) and 1(c)] is necessary when they do gardening, put on socks or clip their toenails.

Recently, some modifications have been made to traditional total knee arthroplasty (TKA) to increase the range of motion (Akagi et al. 2000; Huang et al. 2005; Morra and Greenwald 2005; Nakayama et al. 2005). The Scorpio NRG (Non-Restricted Geometry, Stryker Co., USA) is a good example of the various modifications that attempt to improve the range of motion (Todo et al. 2007). Yet the maximum flexion angle is limited to about $130^{\circ}$, which is not enough for performing a seiza. A new type of knee prosthesis, designated CFK (Complete Flexion Knee, Japan Medical Material Co., Japan) (Lawi et al. 2009), has been developed by our research group to address a better range of motion and durability so as to enable the patient to make a complete knee flexion, in which the maximum knee flexion angle reaches above $150^{\circ}$.

A prosthesis has to balance the range of flexion, intrinsic stability and durability. Thus, our CFK with high stability and mobility has to be further assessed with respect to the durability in addition to the strength. Since the durability of an artificial knee joint is mainly attributed to wear of the polyethylene tibial insert (Puloski et al. 2001), it is essential to focus on determining the principal stress (shear stress) or von Mises stress to predict subsurface delamination wear on the insert. In Japan the law does not allow cadaveric studies, and the specimen cannot be harvested as planned. Thus, we need to perform an alternative such as a finite-element model (FEM) analysis or a photoelastic analysis.

FEM analyses have been extensive for prosthetic stress because they can be applied to the problem of the stressstrain levels induced in the internal parts of the tibial insert (Shashishekar and Ramesh 2007; Todo et al. 2007). However, the results of conventional FEM analyses greatly depend upon the way the meshes are created. Ramesh and Pathak (1999) noted that photoelastic isochromatics can be effectively used to detect FEM meshing problems.

*Corresponding author. Email: hirokawa@mech.kyushu-u.ac.jp 


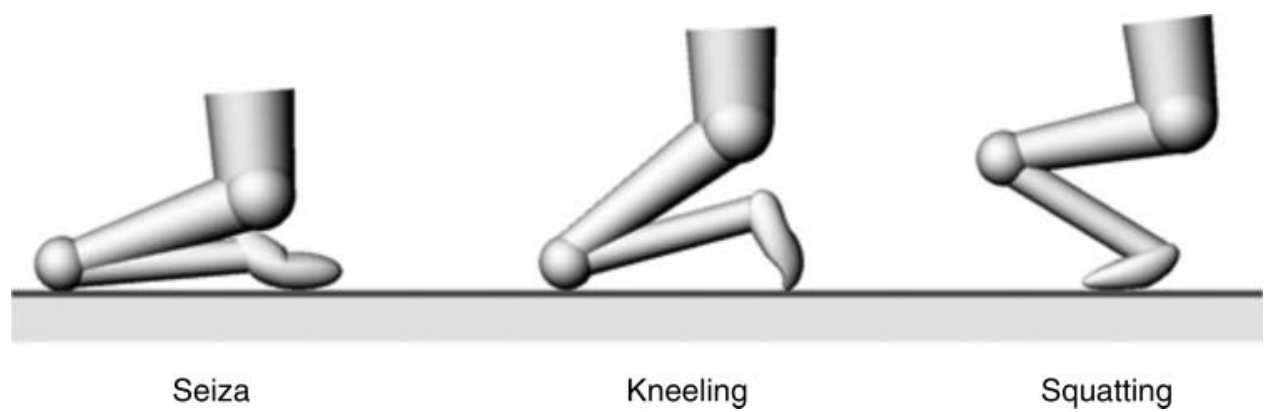

Figure 1. Typical examples of high/deep knee flexion.

For the above reasons, we had previously conducted a photoelastic experiment to evaluate the stress values and distribution on the tibial inserts for Scorpio NRG and CFK (Lawi et al. 2008). Yet in our previous study, we had applied loads with a fixed value to the fixed direction of the prosthetic models. In this study, we intended to apply loads which would duplicate those acting on the knee joint during ascent from deep knee flexion. Then, we faced the most fundamental problem: the information about the forces acting on the joints during deep knee flexion was lacking. The only study was by Dahlkvist et al. (1982), with a limited number of subjects. Recently in vivo data acting on the knee prosthesis have been reported (D'Lima et al. 2005). Still, most of them have been about activities in the low/middle flexion angle. It should be stressed that the data on activities of the knee joint forces in deep flexion are lacking. This was the reason why we had to perform the simulation despite the fact that some in vivo data were already available.

Although the ultimate goal of this study is to determine the stress on the CFK insert during ascending from seiza, a formulation of this motion is quite complex. Thus squatting [Figure 1(c)] was chosen as an example of high/deep knee flexion in this study. We know that seiza, kneeling and squatting each have different requirements on the prosthesis. Yet in Figure 1, ascending from seiza would include the same requirements as those from kneeling and squatting to some extent, and the results of photoelastic stress analysis for squatting may be applicable to the case of seiza.

We introduced the data on the forces acting on the knee joints during ascending from squatting by creating a two-dimensional mathematical model and carrying out a measurement experiment for 12 subjects (Fukunaga and Hirokawa 2009). Then, using our data, we carried out the photoelastic experiment. Furthermore, we performed the FEM analyses under the same conditions as photoelasticity for the purpose of comparison between the two methods, thereby verifying the validity of our photoelastic method.

\section{Materials and methods}

\subsection{Prostheses}

The prostheses used in this study are the Scorpio NRG [Figure 2(a)] and the CFK [Figure 2(b)].

The posterior-stabilised knee prostheses are designed to stabilise anterior-posterior knee motion by mechanical interaction between the tibial post and the femoral cam after removal of the posterior cruciate ligament. A contemporary posterior-stabilised knee prosthesis, NRG, is designed for high flexion by allowing sufficient rollback to avoid bony impingement without increased resection. The tibial insert's articulating surface adapts a spherical arc in order to realise greater rotational freedom. Additionally, its post-and-cam configurations help to accommodate the rotations on the post (Tamaki et al. 2008).

CFK has a unique ball-and-socket joint in the midposterior portion of the femoral and tibial components, which functions as a posterior-stabilising cam mechanism and also as a load-bearing surface. The principal objectives of this prosthesis are to avoid an impingement by offsetting the ball position, to allow internal/external rotation by the spherical bearing surfaces of the post-cam (ball-socket) architecture and to avoid subluxation by keeping continuous contact between the spherical surfaces even at the state of complete knee flexion.
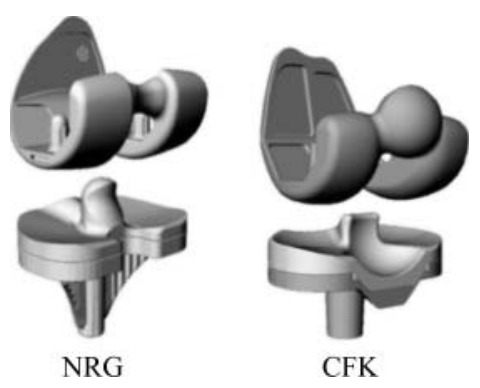

Figure 2. Overviews of two types of prostheses designed to attain high/deep flexion of the knee. 


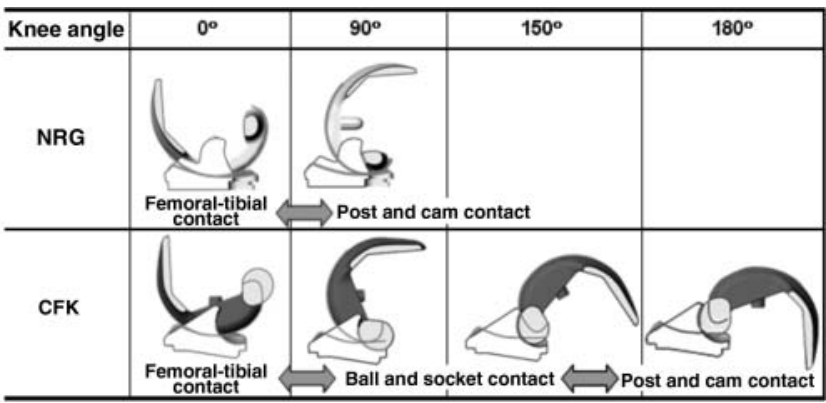

Figure 3. Articulation modalities of the two prostheses according to the knee angle.

Figure 3 shows the articulation modalities of the NRG and CFK, respectively, according to the knee angle. For the NRG, the femoral cam comes into contact with the tibial post at around $60^{\circ}$ of flexion. After that, it has three contact points: the medial and lateral sides of tibio-femoral articulating surfaces and the post and cam. The post and cam are to enhance the rollback but not to bear the load; they cannot function over $\sim 100^{\circ}$ of flexion.

CFK can maintain a contact on either the tibio-femoral articulating surfaces or the ball and socket (post and cam). Here the ball and socket and the post and cam are virtually the same structure. When the knee angle is smaller than $\sim 90^{\circ}$, we call the structure the ball and socket, and after it becomes larger than $90^{\circ}$ we call it the post and cam. The post and cam of CFK functions as a load-bearing joint over $90^{\circ}$ and theoretically up to $180^{\circ}$ of knee flexion.

\subsection{Frozen-stress photoelasticity}

Photoelastic measurement relies on the fact that the model being studied, when under stresses, can split an incident light vector into two components (Hirokawa et al. 1998). The two components develop phase shifts of the light wave, and their relative retardation is proportional to the difference between the two principal-stress values. When a model is viewed in monochromatic light, the relative retardation is counted as the fringe order. For linear photoelastic material, the difference in the principal strains can be measured by establishing the fringe order as well. This relation is expressed as

$$
\frac{\sigma_{1}-\sigma_{2}}{2}=\frac{n f_{s}}{t} \quad\left(N / m^{2}\right)
$$

where $\left(\sigma_{1}-\sigma_{2}\right) / 2=$ maximum shear stress $\left(\mathrm{N} / \mathrm{m}^{2}\right) ; n$ is the fringe order at the point in question; $f_{s}$ is the material fringe value $\left(\mathrm{N} / \mathrm{m}^{2} /\right.$ fringe $\left./ \mathrm{m}\right)$; and $t$ is the thickness $(\mathrm{m})$.

Stress-freezing photoelastic analysis is based on a behaviour that is exhibited by polymeric substances when they are heated. When a high-polymer material is heated to a temperature slightly above its softening point, or its critical temperature (glass-transition temperature), its rigidity, expressed, for example, by the modulus elasticity, is greatly reduced, although the material retains its elastic behaviour. Suppose a model is loaded at this temperature and then the applied loads are retained; while the model is cooled to ambient temperature, a state of stress remains 'frozen' in the model (Theocaris and Gdoutos 1979). After stress freezing, the model is sectioned into slices without disturbing the stress field, and then the stress in each slice is analysed using two-dimensional methods. For the analyses of the frozen-stress fringe pattern, it is necessary to know the material fringe value of the model material at the stress-freezing temperature (Stewart and Edwards 1984).

In this study, we fabricated the tibial insert models with epoxy monomer by mixing Craft Resin Z-1 araldite and hardener (Nisshin Resin Co. Ltd., Japan). With the same monomer as the insert model, we fabricated a calibration beam in order to introduce the glass-transition temperature of the model material as well as to introduce the material fringe value for strain.

\subsection{Loading condition and experimental set-up}

\subsubsection{Loading condition}

As already mentioned, we introduced the data on the forces acting on the knee joints during ascending from deep squatting, using a two-dimensional mathematical model of a lower limb as shown in Figure 4, which we created referring to Dahlkvist et al.'s (1982) study. Since the structure and condition of our model have been reported in the literature (Fukunaga and Hirokawa 2009), they will be explained only briefly here.

We denoted the moment about the hip joint as $M_{h}$, about the knee joint as $M_{k}$ and about the ankle joint as $M_{a}$.

In the following equations, the symbols $a, b$ and $c$ stand for the lengths of the moment arm about the hip, the knee and the ankle joints respectively. For example, $a_{Z}$ stands for the moment arm of $Z$ (floor-reacting force) about the hip joint.

The moment about the hip joint by external forces is expressed as

$$
M_{\mathrm{h}}=Z a_{Z}+X a_{X}-W_{1} a_{W 1}-W_{2} a_{W 2}-W_{3} a_{W 3} .
$$

The moment about the hip joint by external forces may also be expressed as

$$
M_{\mathrm{h}}=\mathrm{GM} a_{\mathrm{GM}}+H a_{H}-R a_{R}+N a_{N} .
$$

Since the values from Equations (2) and (3) must be identical to each other, the following equation holds: 


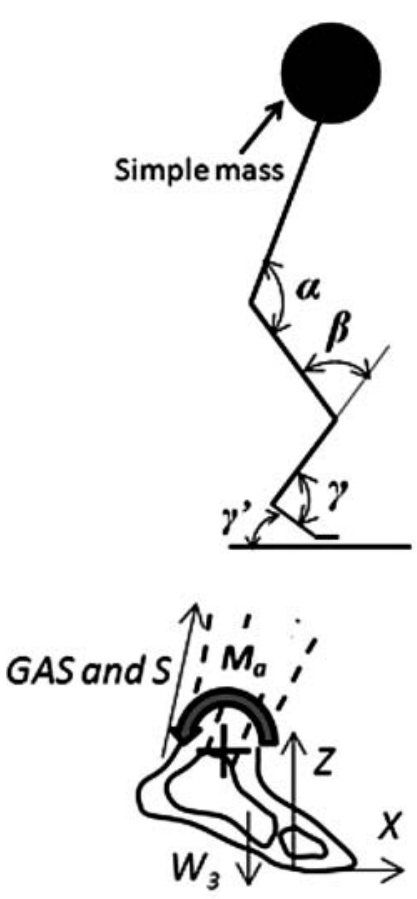

Around Ankle Joint

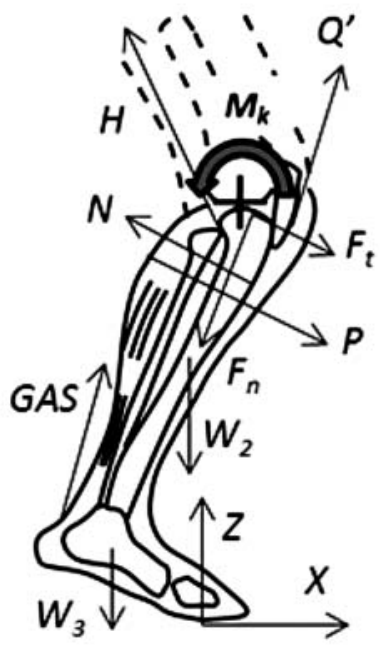

Around Knee Joint

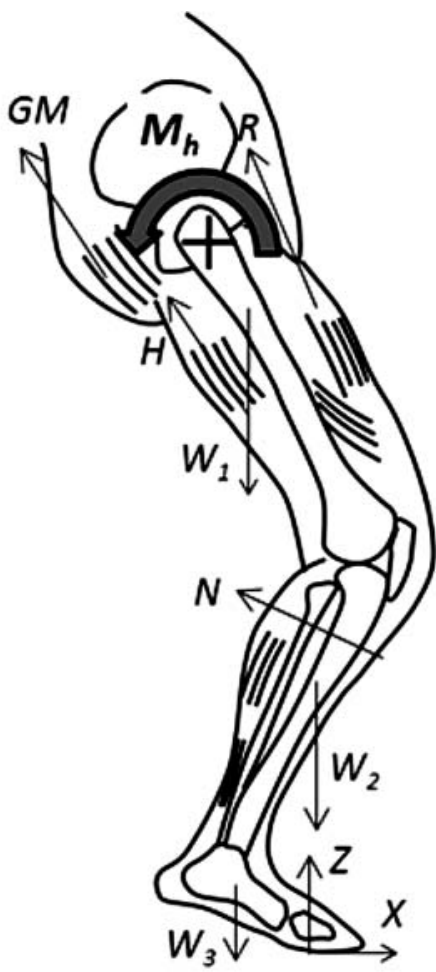

Around Hip Joint

Figure 4. A two-dimensional model of a lower limb and definition of the joint angles. The forces for calculating the moment equilibrium condition around each joint are as follows. Tensile force of the muscle/tendon: $H=$ hamstrings, GM $=$ gluteus, $R=$ rectus femoris, GAS $=$ gastrocnemius, $S=$ soleus and $Q^{\prime}=$ patella tendon. Forces acting on the knee joint: $F_{n}=$ the normal component, which is parallel to the tibial axis, and $F_{t}=$ the tangential component. External forces: $W=$ the gravity force, $Z$ and $X=$ the normal and the tangential component of the floor-reacting force and $N=$ the normal component of the floor-reacting force during kneeling.

$$
\begin{aligned}
Z a_{Z} & +X a_{X}-W_{1} a_{W 1}-W_{2} a_{W 2}-W_{3} a_{W 3}-\mathrm{GM} a_{\mathrm{GM}} \\
& +H a_{H}-R a_{R}+N a_{N}=0 .
\end{aligned}
$$

In the same way as the above, the equations for the knee and ankle joints are introduced as the following equations respectively:

$$
\begin{aligned}
-Z b_{Z} & +X b_{X}+W_{2} b_{W 2}+W_{3} b_{W 3}-\mathrm{GASb}_{G A S}+Q^{\prime} b_{Q^{\prime}} \\
& -H b_{H}-N b_{N}+P b_{P}=0
\end{aligned}
$$

and

$$
Z c_{Z}+X c_{X}-W_{3} a_{W 3}-(\mathrm{GAS}+S) c_{\mathrm{GAS}}=0
$$

From Equations (4)-(6), the number of unknowns is six: GM, $H, R$, GAS, $Q^{\prime}$ and $S$. In order to solve this statically indeterminate equation, the following three assumptions are to be employed:

1. Hamstrings and gluteus work synchronously.

2. Gastrocnemius and soleus work synchronously.

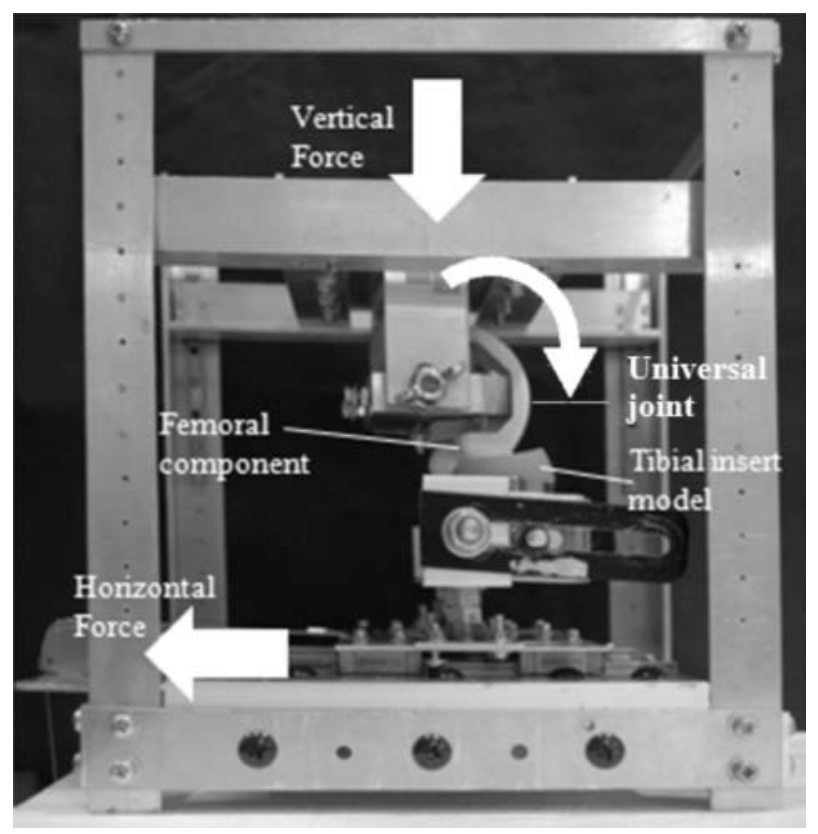

Figure 5. Experimental set-up. 


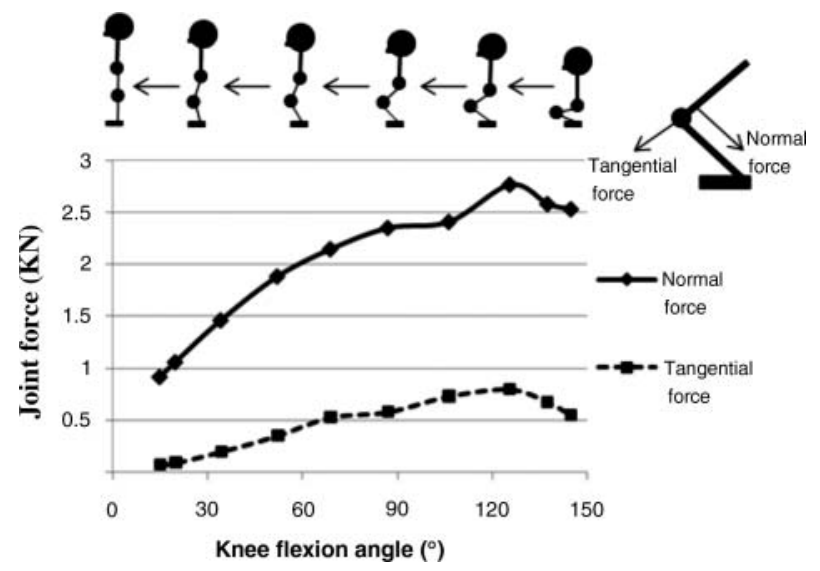

Figure 6. Variation of the knee joint forces as a function of the knee angle when ascending from squatting.

3. The ratio between the tension of patella tendon and the force of the quadriceps is the function of the knee flexion angle (van Eijden et al. 1988).

From Equations (4)-(6) and the above-mentioned three assumptions, we are able to introduce the muscle forces acting on the joints and consequently the forces acting on the knee joint, by inputting the measurement data in terms of joints' angles and floor reaction forces during the motion into the equations.

First some measurement experiments for low/middle knee flexion motion were carried out to obtain the abovementioned measurement data. Then we compared the results with those from the literature such as those from in vivo measurements for level walking and stair climbing (Andriacchi et al. 1982) and those from simulations for level walking (Morrison et al. 1970) and rising from a chair (Ellis et al. 1984), thereby verifying the validity of our results. Next we performed the measurement experiment for ascending from squatting and introduced the knee joint forces during the motion; they were used as the loading condition for the following photoelastic experiment.

\subsubsection{Experimental set-up}

A special tool was designed to permit identical simultaneous loading to the model in a temperature-controlled environmental chamber (Figure 5). A universal joint was used

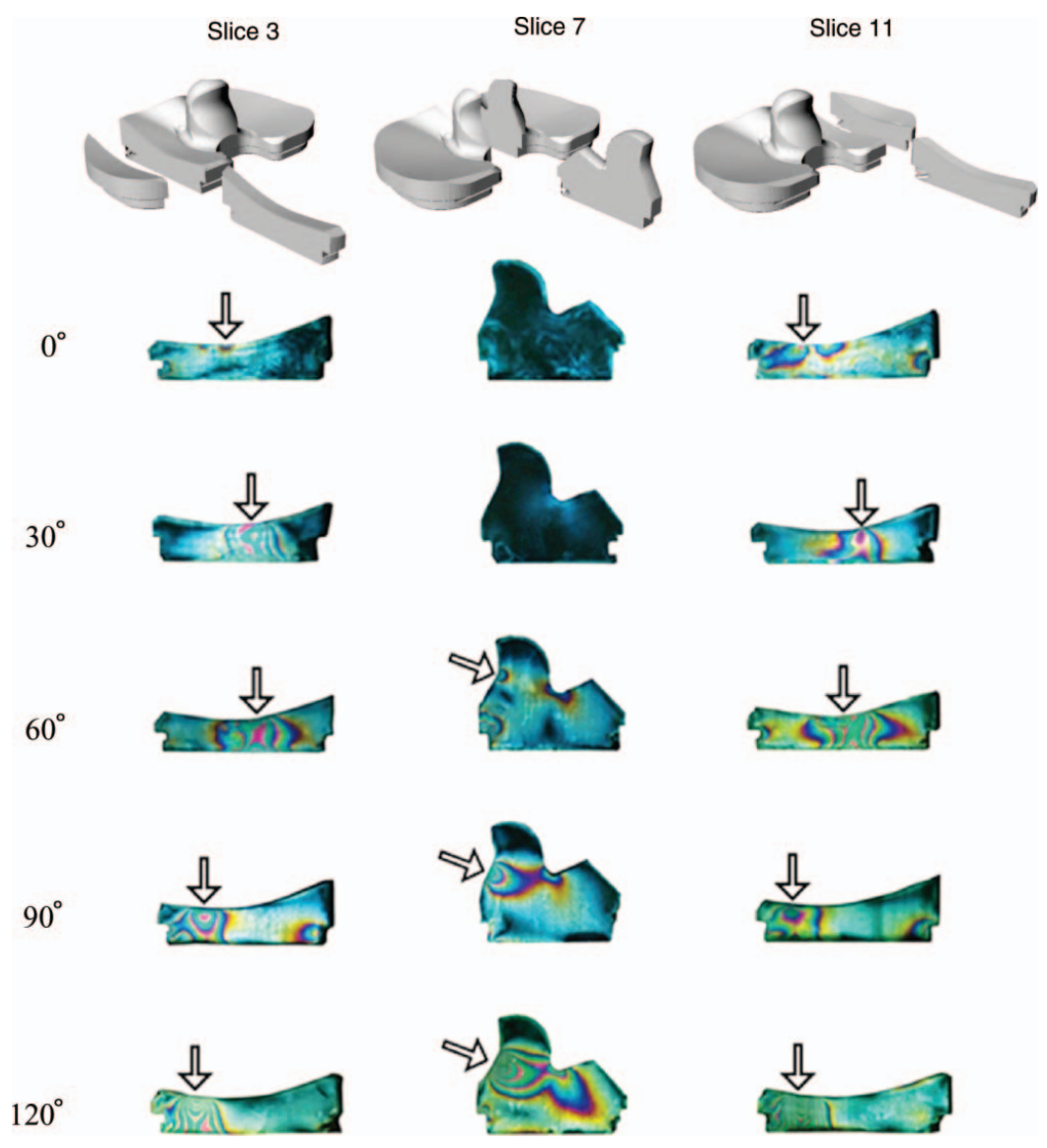

Figure 7. Isochromatics on slice 3 (medial), slice 7 (mid-posterior) and slice 11 (lateral) of the tibial insert of NRG (an arrow points the stress concentrating portion, same as Figure 8). 
to set the tibia with arbitral three-dimensional orientations relative to the femur, thereby making knee flexion angles.

Since the epoxy model has a known elastic modulus at stress-freezing temperatures, an appropriate load scaling was applied according to the joint force in each angle. We divided the values of the normal and tangential forces (Figure 6) by 50 and applied them as the vertical and horizontal forces respectively to the loading apparatus.

Under the loads that were being applied to the model, the loading apparatus with the model was put into a temperature-controlled environmental chamber and kept at $50^{\circ} \mathrm{C}$ overnight. Then the loading apparatus with the model was cooled down to room temperature, and the model under a stress-freezing state was obtained.

For the purpose of measuring photoelastic fringes, the model was cut into 13 slices along the sagittal direction (slice 1 was the lateral-most slice and slice 13 was the medial-most one). The photoelastic stress analyses were done on the basis of the fringe patterns and their colours.

\subsection{Comparison with FEM analysis}

We performed the FEM analysis to verify the validity of the results from our photoelastic experiments. The commercially available FEM software package (LS-DYNA PC version 971, JSOL Co., Japan) was employed for analysis. We set the material properties of the models and the loading condition with the same values as in the experiment. The numbers of the nodes and the elements were 38,179 and 121,604 for the NRG model and 21,958 and 89,322 for the CFK model respectively.

\section{Results}

Figures 7 and 8 show the isochromatics of the typical three areas at various knee flexion angles for NRG and CFK respectively. The small arrows indicate stress concentrating points. The typical three areas to be analysed were the medial (slice 3), the mid-posterior (slice 7) and the lateral area (slice 11). The mid-posterior area was selected mainly

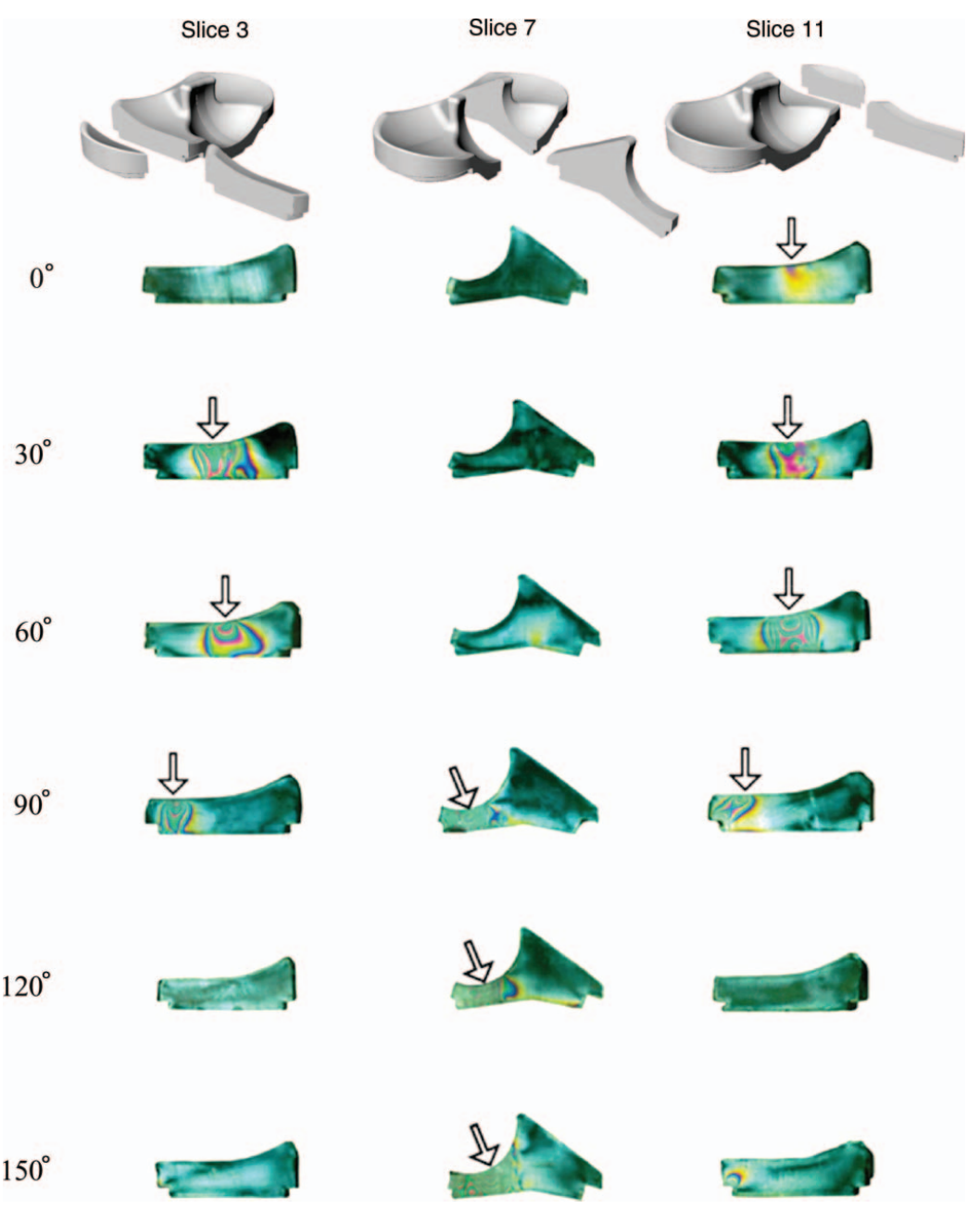

Figure 8. Isochromatics on slice 3 (medial), slice 7 (mid-posterior) and slice 11 (lateral) of the tibial insert of CFK. 


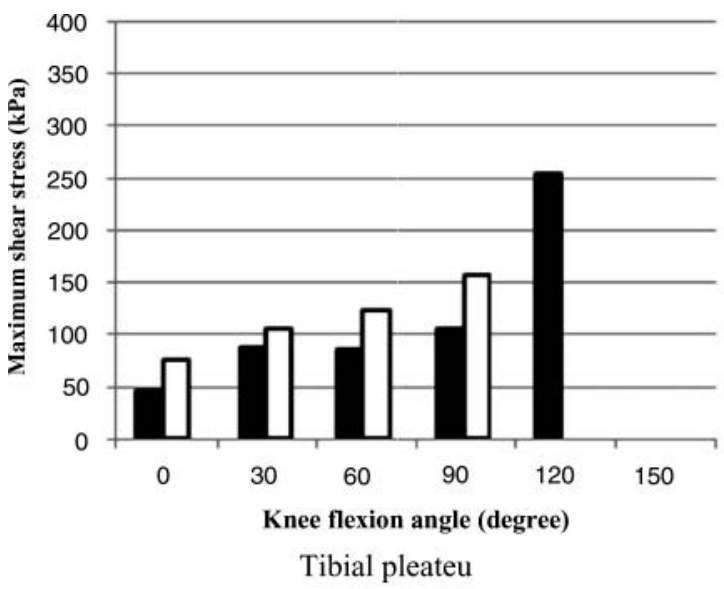

NRG tibial plateau

口CEK tibial plateau

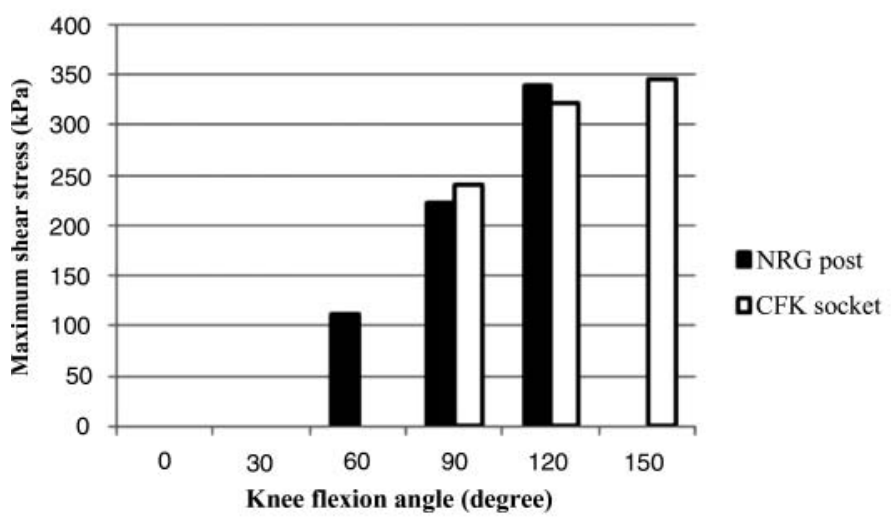

Post or socket

Figure 9. Comparison of the maximum shear stresses for the NRG and CFK models as a function of the knee angle.

to assess the stress on the post (socket) at high/deep knee flexion, while the medial and lateral areas were selected to assess the stress on the femoral condylar articulating surface of the insert at low/middle knee flexion.

By comparing Figures 7 and 8, we found the following phenomena. For the NRG, a post-cam contact initiates at $60^{\circ}$ of knee angle, and after that the contacts are seen on three slices, which continue until $120^{\circ}$. In our loading apparatus and loading condition, the post and the cam could not make a contact at $150^{\circ}$ of knee flexion.

For the CFK, the three points' contact occurs at $90^{\circ}$ of knee angle. After that only a post-cam (ball-socket) contact continues until $150^{\circ}$, with no contact on either the medial or the lateral area.

It is seen that the stressed area is wider for the NRG. At $30^{\circ}, 60^{\circ}$ and $90^{\circ}$, stress concentrating areas are similar for both NRG and CFK. Stress concentrating points move to the posterior side according to the knee angle, indicating a rollback of the femoral condyle on the tibial plateau.

The stress concentrating point of the CFK in the midposterior is lower as compared to the NRG, which means that the 'jump height' of the CFK is enough to prevent the femur's disengagement even at deep flexion.
Figures 9(a) and 9(b) show the maximum shear stresses on the tibial plateau and on the post or socket portions for the NRG and CFK respectively as a function of the knee angles. The stresses on the tibial plateau are the mean values of stresses on the medial and lateral plateaus. Both Figures 9(a) and 9(b) show significant increase in stresses in accordance with the knee flexion angle. Figure 9(a) shows that the stress on the tibial plateau is lower for the NRG than for the CFK from $0^{\circ}$ to $90^{\circ}$. Figure 9 (b) shows that the stress on the NRG's post is lower than that on the CFK's socket at $90^{\circ}$, whose relation is reversed at $120^{\circ}$ despite the fact that the CFK does not make contact on the tibial plateau. This is because the CFK's ball and socket make a convex-concave contact.

Figures 10(a) and 10(b) illustrate the variations of maximum shear stresses for the NRG and CFK respectively as functions of the knee angles; the results from the FEM analyses are also superimposed. Overall, good agreement is found between the photoelastic experiments and the FEM analyses. In Figure 10(b), we see large differences in maximum shear stresses on the socket between the photoelasticity and the FEM. This is because the contact points are slightly different from each other, indicating instability of contact modality for the CFK's ball and socket. 

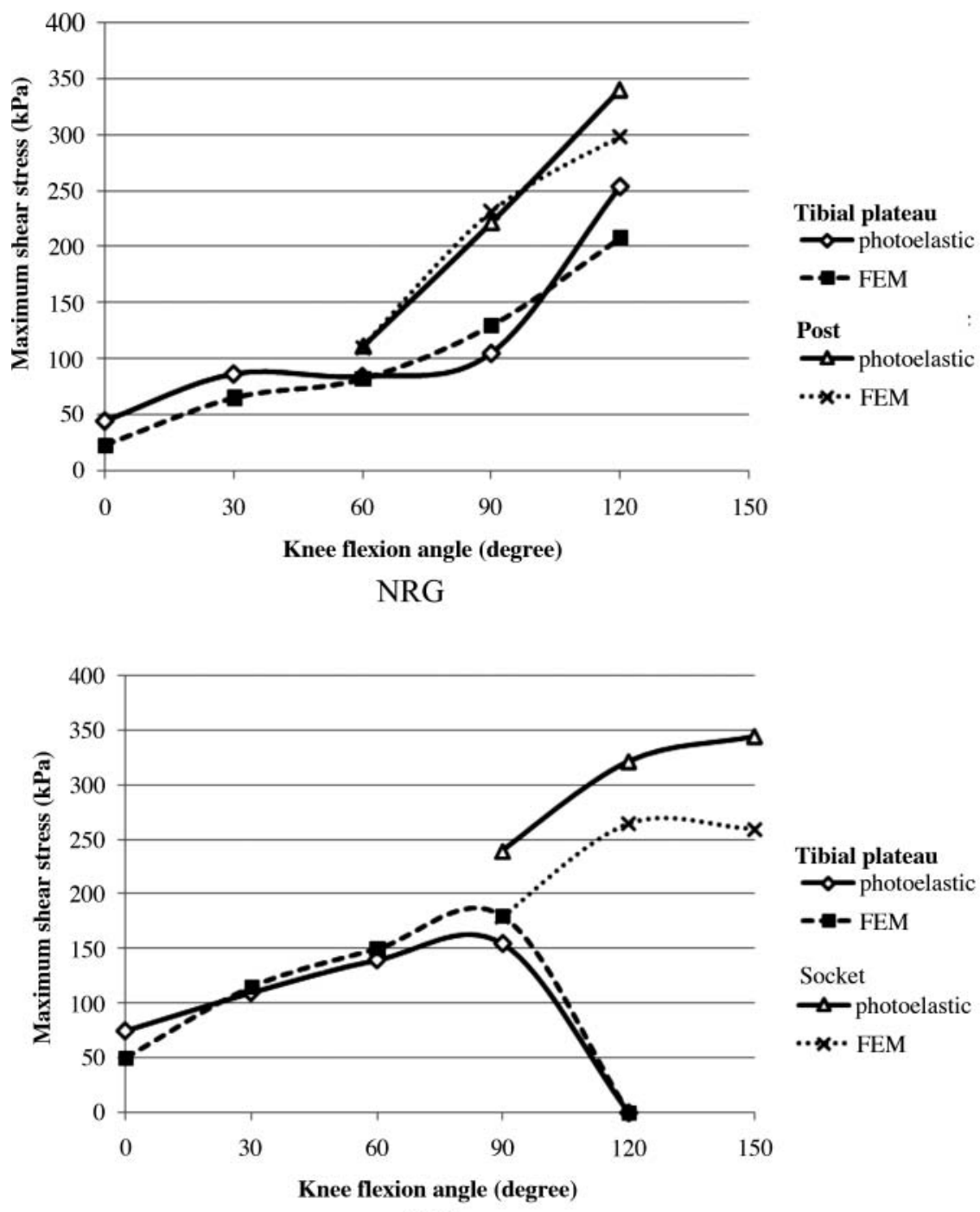

CFK

Figure 10. Comparison of the maximum shear stresses from the photoelasticity and the FEM as a function of the knee angle.

\section{Discussion}

A prosthesis has to balance the range of flexion, intrinsic stability and durability. Thus our CFK with high stability and mobility has to be further assessed with respect to durability. Since the durability of a prosthesis is mainly attributed to wear of the polyethylene insert, it is essential to focus on determining the magnitude and distribution of stresses on the insert.

The following counter-arguments may arise against the loading condition in association with the knee angle used in our photoelastic experiment. First, when we squat or sit in a seiza style, the tibio-femoral contact force is negligible because the tibio-femoral surfaces are almost separate. Thus it is impractical to apply such large force values as those shown in Figure 6 at deep knee flexion. Next, most prosthetic patients cannot or should not be allowed to per- form such a severe motion with the knee joints as the ones shown in Figure 6.

The answers to the above are as follows. First, since a TKA knee does not have the cruciate ligaments, after both the components are separate, the knee may cause subluxation when flexing. Thus continuous contact between the tibial and femoral components has to be maintained, and consequently contact forces between the post and cam or the ball and socket are to be generated. Next, it should be noted that sometimes excessively high loads, the same as shown in Figure 6, could be applied to the prosthesis no matter how gently the patient may rise from seiza. And from the risk analysis viewpoint, it is necessary to check the strength of the polyethylene insert under an extreme loading condition as given in Figure 6. As already mentioned in the introduction, we believe that ascending from 
seiza would be similar to ascending from squatting and that the results from squatting can be applied to seiza.

Nakagawa et al. (2000) reported that for a normal Japanese knee $90^{\circ}-162^{\circ}$ of knee flexion is accompanied by about $28^{\circ}$ of tibial internal rotation. This amount of rotation combined with stability in high flexion has so far been possible in the modern TKA designs only through the Scorpio NRG. The post and cam of the Scorpio NRG have a round-on-round design which can produce axial rotation of approximately $25^{\circ}$ during the deep knee-bending motion (Tamaki et al. 2008). However, Akasaki et al. (2008) found that this design feature could generate high values of contact stress due to smaller contact area between the post and the cam in comparison with other prostheses. It is reported that high contact stress results in an increased amount of polyethylene wear in knee wear simulators (Miura et al. 2002).

The trade-off between the ranges of flexion follows by axial rotation, and consideration of reduction of wear of tibial post to increase its longevity during high flexion has been a great challenge until now in modern TKA.

The CFK prosthesis presents a unique design for achieving deep flexion without affecting the durability of the prosthesis. The cam mechanism is designed to work not only as a femoral rollback driver or a posterior stabiliser but also as a weight-bearing surface, particularly during deep flexion. An idea similar to the CFK prosthesis for load-bearing surface in deep flexion, namely the bi-surface knee prosthesis (Japan Medical Material Co., Japan), has been introduced by Akagi et al. (2000). However, the bi-surface prosthesis cannot completely satisfy its design objectives for deep flexion and long-term survival. It was found at a mean follow-up period of 6 years that $20 \%$ users felt looseness in their knee and $2 \%$ required revision for instability (Akagi 2005). The CFK prosthesis recovers the fundamental concept by modifying the ball-and-socket joint and reshaping the geometry. In addition, same as the NRG design of round-on-round post and cam, the CFK reproduces a great rotational freedom of the femur relative to the tibia in deep flexion.

The results from our experiment demonstrated that the stress on the tibial plateau(s) was relatively low and that its variation pattern was uniform for the NRG while the knee flexion angle was smaller than $90^{\circ}$ [Figures 9(a) and 10(a)]. This means that the configuration between the femoral condyle and the tibial plateau of the NRG is well designed for the usual activities of the lower limbs, such as walking. Unfortunately however, the NRG does not introduce stress on the post when the knee angle is larger than $120^{\circ}$ [Figures 7, 9(a) and 10(a)], indicating that it has not been designed to make a knee flexion angle larger than $120^{\circ}$. For a range of knee flexion angle between $90^{\circ}$ and $120^{\circ}$, stress values on the post are relatively high despite the contact between the femoral condyle and the tibial plateau also being maintained. This means that the post-cam configurations are not well designed from a weight-bearing viewpoint. As for the
CFK, the contact is only on the ball and socket after $90^{\circ}$ of knee flexion. Considering this situation, the stresses on the socket are not high, demonstrating the spherical-bearing structure of the ball and socket to be an advantage.

There are limitations in this study. The internal tibial rotation did not apply because our experimental apparatus could not make such motion. To overcome this problem, we are planning to develop our loading jig to assess the complete knee kinematic system.

\section{Conclusion}

We performed photoelastic studies to determine the magnitude and distribution of stresses on the polyethylene insert of two types of posterior-stabilised knee prostheses which had been designed to attain high/deep knee flexion. They were the NRG (a conventional posterior-stabilising prosthesis) and the CFK (a newly designed prosthesis aiming to make a complete knee flexion such as seiza). We chose ascending from squatting as our study subject instead of ascending from seiza.

While the knee angle was smaller than $90^{\circ}$, contacts were made on the tibio-femoral articulating surfaces. The results demonstrated that the NRG has high conformity with low stress. After the knee angle became larger than $90^{\circ}$, a contact was made on the post and cam for the NRG and on the ball and socket for the CFK. Thus the stresses on the mid-posterior slice were mainly investigated. The results demonstrated that the CFK has high conformity with lowest stress. Over about $120^{\circ}$ of knee flexion, it was found that the post-cam contact could not be maintained for the NRG. We therefore conclude that the CFK has adequate configuration for deep knee flexion from a load-bearing viewpoint.

Conflict of Interest Statement: The authors find no conflict of interest related to this paper and its publication in Applied Bionics and Biomechanics.

\section{Acknowledgements}

This research programme is supported by the Japanese Government Grant in Aid for General Scientific Research, category B(1) (2009, 20300161).

\section{References}

Akagi M. 2005. Deep knee flexion in the Asian population. Total Knee Arthro. VI:311-316.

Akagi M, Nakamura T, Matsusue Y, Ueo T, Nishijyo K, Ohnishi E. 2000. The bisurface total knee replacement: a unique design for flexion. J Bone Joint Surg. 82-A:1626-1633.

Akasaki Y, Matsuda S, Shimoto T, Miura H, Higaki H, Iwamoto Y. 2008. Contact stress analysis of the conforming post-cam mechanism in posterior-stabilized total knee arthroplasty. J Arthro. 23:736-743.

Andriacchi TP, Galante JO, Fermier RW. 1982. The influence of total knee-replacement design on walking and stair climbing. J Bone Joint Surg Am. 64:1328-1335. 
D’Lima DD, Patil S, Steklov N, Slamin J, Colwell C. 2005. Tibial forces measured in vivo after total knee arthroplasty. J Arthro. 21:255-262.

Dahlkvist NJ, Mayo P, Seedhom BB. 1982. Forces during squatting and rising from a deep squat. Eng Med. 11:69-76.

Ellis MI, Seedhom BB, Wright V. 1984. Forces in the knee joint whilst rising from a seated position. J Biomed Eng. 6:113120.

Fukunaga M, Hirokawa S. 2009. The model analysis of lower limb at ascending from deep knee flexion. Vol 69. Memoires of the Faculty of Engineering, Kyushu University, Fukuoka, Japan, 139-148.

Hirokawa S, Yamamoto K, Kawada T. 1998. A photoelastic study of ligament strain. IEEE Trans Rehab Eng. 6:300-308.

Huang H, Su JY, Wang G. 2005. The early results of high-flex total knee arthroplasty: a minimum of 2 years of follow-up. J Arthro. 20:120-1244.

Lawi A, Sekiya K, Takiguchi J, Hirokawa S. 2008. A photoelastic study of contact stress on the tibial insert of knee prosthesis at deep flexion. Vol 68. Memoirs of the Faculty of Engineering, Kyushu University, Fukuoka, Japan, 141-150.

Lawi A, Sekiya K, Takiguchi J, Hirokawa S. 2009. Polymer insert stress in knee prosthesis design at high flexion: a photoelastic study. Proceedings of the 55th Conference of Orthopaedic Research Society, Las Vegas, USA, CD (Paper No. 2080).

Miura H, Higaki H, Nakanishi Y, Mawatari T, Morooka T, Murakami T, Iwamoto Y. 2002. Prediction of total knee arthroplasty ployethylene wear using the wear index. J Arthro. 17:760-766.

Morra EA, Greenwald AS. 2005. Polymer insert stress in total knee designs during high-flexion activities: a finite element study. J Bone Joint Surg. 87-B:120-124.

Morrison JB, et al. 1970. The mechanics of the knee joint in relation to normal walking. J Biomech. 3:51-61.
Nakagawa S, Kadoya Y, Todo S, Kobayashi A, Sakamoto H, Freeman MA, Yamano Y. 2000. Tibiofemoral movement 3: full flexion in the living knee studied by MRI. J Bone Joint Surg. 82-B:1199-1200.

Nakayama K, Matsuda S, Miura H, Higaki H, Otsuka K, Iwamoto H. 2005. Contact stress at the post-cam mechanism in posterior-stabilised total knee arthroplasty. J Bone Joint Surg. 87-B:483-488.

Puloski SKT, McCalden RW, et al. 2001. Tibial post in posterior stabilized total knee arthroplasty. J Bone Joint Surg. 83A:390-397.

Ramesh K, Pathak PM. 1999. Role of the photoelasticity in evolving discretization schemes for FEA analysis. J Exp Tech. 23:36-38.

Shashishekar C, Ramesh CS. 2007. Finite element analysis of prosthetic knee joint using ANSYS. Biomed Health. 12:6572.

Stewart BL, Edwards RO. 1984. Removable partial denture design: a photoelastic study. J Biomed Mater Res. 18:979-990.

Tamaki M, Tomita T, Yamazaki T, Hozack WJ, Yoshikawa H, Sugamoto K. 2008. In vivo kinematic analysis of a highflexion posterior stabilized fixed-bearing knee prosthesis in deep knee-bending motion. J Arthro. 23:879-885.

Thambyah A, Goh JCH, Das De S. 2005. Contact stresess in the knee joint in deep flexion. Med Eng Phys. 27:329-335.

Theocaris PS, Gdoutos EE. 1979. Matrix theory of photoelasticity. Springer series in optical sciences. Vol 11. Berlin Heidelberg: Springer-Verlag. 132-137.

Todo M, Nagamine R, Yamaguchi S. 2007. Stress analysis of PS type knee prostheses under deep flexion. J Biomech Sci Eng. 2:237-245.

van Eijden TMGJ, Kouwenhoven E, Verburg J, Weijs WA. 1986. A mathematical model of the patellar femoral joint. J Biomech. 19:219-229. 

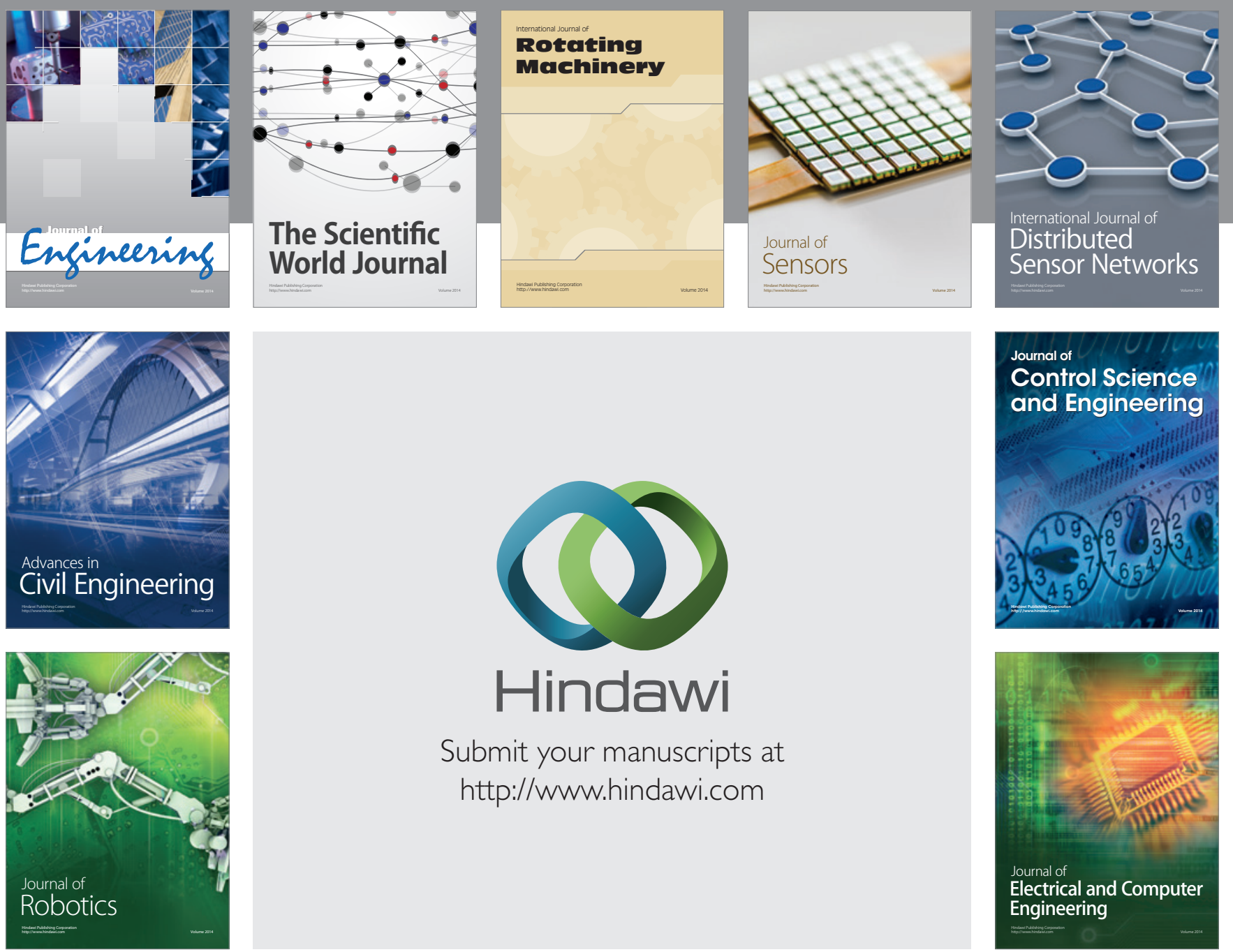

Submit your manuscripts at

http://www.hindawi.com
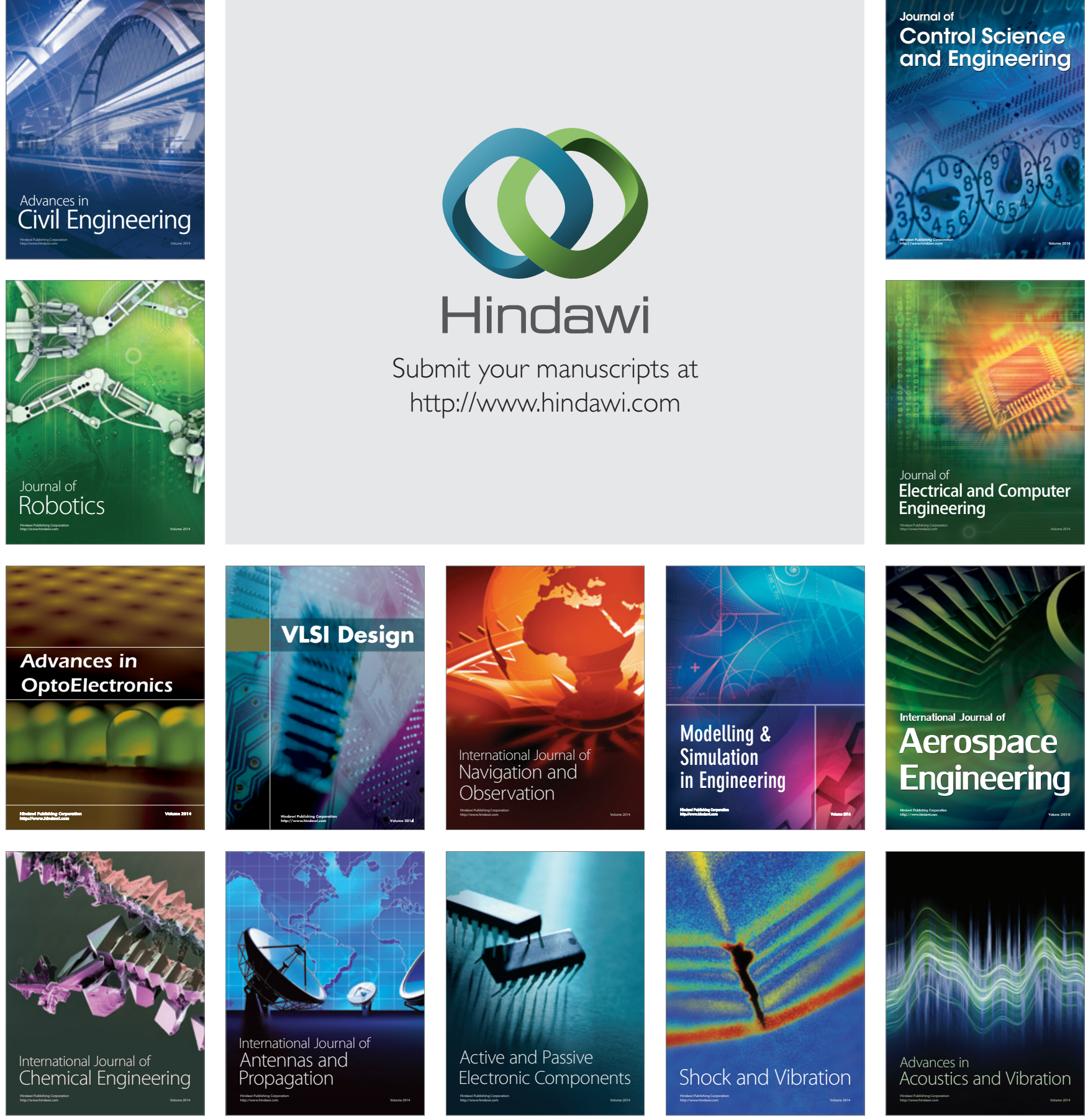